\title{
Grundlegende Erkenntnisse im Rahmen des Pilotprojekt Bad Deutsch-Altenburg - Die Bedeutung der Stromsohle und Uferzonen für ökologische Prozesse und Artengemeinschaften an einem stark regulierten Fluss, der Donau
}

\author{
Elisabeth Bondar-Kunze · Marina Ivankovic · Andrea Funk · Daniel Trauner · Hubert Keckeis · Thomas Hein
}

Online publiziert: 24. März 2016

(c) Die Autor(en) 2016. Dieser Artikel ist auf Springerlink.com mit Open Access verfügbar.

\begin{abstract}
Zusammenfassung Flüsse benötigen für eine langfristige ökologische Stabilität - Bereiche, die bei verschiedenen Abflusssituationen als hoch produktive Lebensräume verfügbar sind und entsprechend mit dem Fluss in Austausch stehen. Diese Retentionsbereiche oder bioaktive Zonen sind wichtig für die flusseigene Tierwelt als Refugialräume (z. B. Makrozoobenthos und Fische) oder bestimmte Entwicklungsstadien (z. B. Jungfische), dienen benthischen Algengemeinschaften zum Aufbau von Biomasse und sind damit Grundlage für das Nahrungsnetz des Flusses sowie den Stoffrückhalt und Umbau (z. B. Nährstoffaufnahme). Vor allem während Niederwasser sind die Uferbereiche und die Stromsohle selbst von wesentlicher Bedeutung, aber in ihrer Ausprägung durch Regulierungsmaßnahmen deutlich eingeschränkt. Ein grundlegendes Verständnis der Abläufe und funktionalen Zusammenhänge ist erforderlich, damit im Rahmen von wasserbaulichen Maßnahmen negative
\end{abstract}

Mag. E. Bondar-Kunze ( $\square)$.

M. Ivankovic, MSc. • Assoc. Prof. Dr. T. Hein

Universität für Bodenkultur Wien,

Institut für Hydrobiologie und

Gewässermanagement,

Max-Emanuel-Straße 17,

Wien 1180, Österreich

E-Mail: elisabeth.bondar@boku.ac.at

D. Trauner, MSc. · Mag. Dr. A. Funk · Mag. E. Bondar-Kunze · Assoc. Prof.

Dr. T. Hein

WasserCluster Lunz - Biologische Station

$\mathrm{GmbH}$, Dr.-Carl-Kupelwieser-Promenade 5, 3293 Lunz am See, Österreich

ao.Univ.-Prof. Dr. H. Keckeis

Department für Limnologie und

Ozeanographie, Universität Wien,

Wien, Österreich
Konsequenzen hintangehalten werden können bzw. der ökologische Zustand wieder verbessert werden kann. Im Rahmen des Pilotprojekts Bad DeutschAltenburg an der Donau östlich von Wien ist daher nicht nur ein Monitoring der flussbaulichen Maßnahmenumsetzung erforderlich, sondern auch ein wissenschaftlicher Erkenntnisgewinn über grundlegende Zusammenhänge und biologische Abläufe, um optimierte Maßnahmen zum Einsatz bringen zu können. In diesem Artikel werden Ergebnisse mehrjähriger Forschungsarbeiten in den Tiefenzonen der Donau und typischen Uferzonenlebensräumen für die Organismengruppen benthische Algen, Makrozoobenthos und Fische präsentiert.

Sclüsselwörter Regulierter Fluss . Benthische Algen · Makrozoobenthos . Fische · Flussbauliche Maßnahmen . Retentionsräume

First findings in the framework of the pilot project Bad DeutschAltenburg - the relevance of river bed and river shoreline zones in a regulated river (Danube River) for basic ecological processes and riverine communities

Abstract To ensure a long-term ecological stability, rivers need areas that are available at different discharge situations as highly productive habitats which are connected with the river. These retention areas or biologically active zones are important for the riverine communities as refuge (e.g. benthic macroinvertebrates and fish) or the development of benthic algae communities as basis for the riverine food web as well as retention zones to perform various ecosystem services (e.g. nutrient retention). Especially during low water level situations, the river shorelines and the river bed itself are of significant importance, but highly degraded in intensely regulated river systems such as the Danube River. A basic understanding of the processes and functional correlations is required to avoid negative consequences of engineering measures applied and to improve the ecological conditions. In the framework of the pilot project Bad Deutsch-Altenburg in the Danube River east of Vienna not only monitoring the implementation of engineering measures is required, but also scientific knowledge gained about basic ecological relationships and potential responses of riverine communities. This article presents results of several years of investigations on the distribution and development of three organism groups (benthic algae, benthic macroinvertebrates and fish) along different riparian structures and in the river bed of the Danube River.

\section{Einleitung}

Fließgewässer werden oft als Lebensadern der Landschaft betrachtet. Diese haben nicht nur eine Bedeutung als Lebensraum für die Pflanzen- und Tierwelt, sondern waren auch für die Entwicklung der menschlichen Gesellschaft sehr früh bedeutend (Besiedelungsrouten, Niederlassungen). Flüsse bieten für den Menschen vielfältige Nutzungsmöglichkeiten (= Ökosystemdienstleistungen), wie zum Beispiel Trinkwasserversorgung, Energiegewinnung, Nahrungsmittelproduktion und Transport (Schifffahrt) (Winiwarter et al. 2013). Diese intensive Nutzung führt allerdings weltweit $\mathrm{zu}$ anthropogenen Veränderungen der hydromorphologischen Bedingungen 


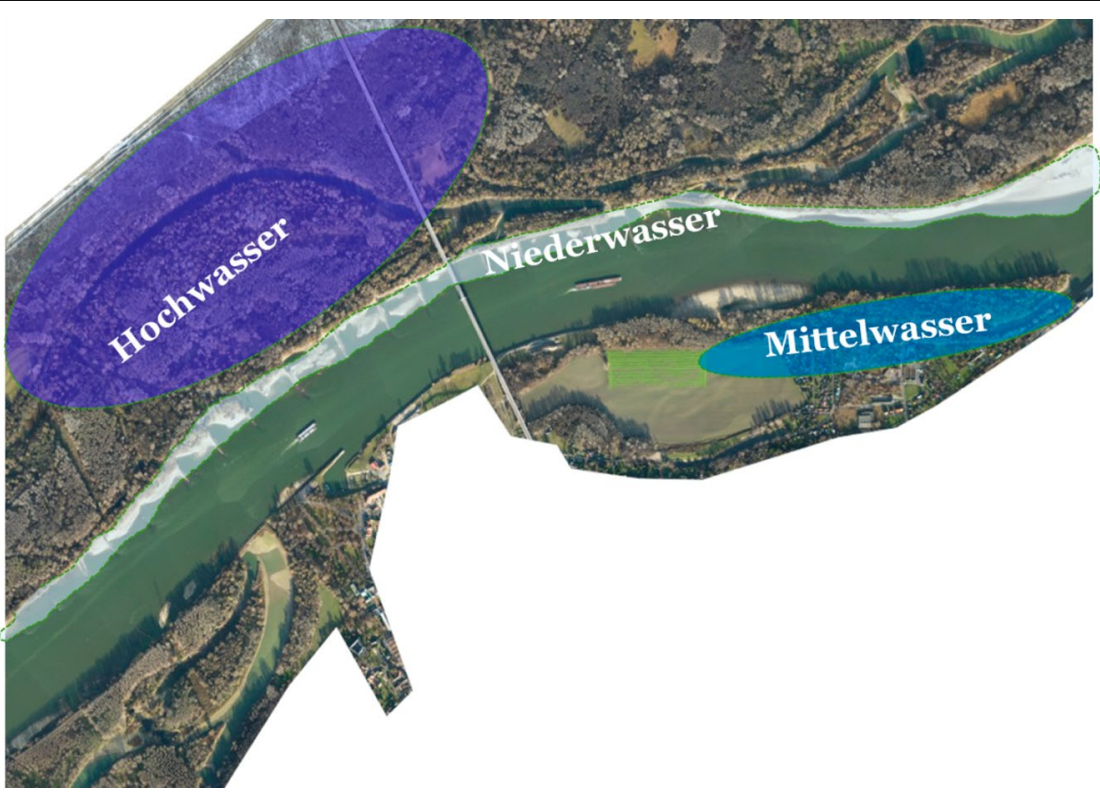

Abb. 1 Darstellung der bioaktiven Zonen/Retentionsbereiche der Donau innerhalb des Projektgebiets. Während Hochwasserphasen ist das Gebiet der Stopfenreuther $\mathrm{Au}$ ein wesentlicher Retentionsbereich, während Mittelwassersituationen kommt Nebengewässern wie dem wiederangebundenen Johler Arm diese Bedeutung zu, und während Niederwasser sind vor allem die Uferbereiche im Hautpgerinne entscheidende Landschaftskomponenten (Satellitenbild (C) Google Earth)

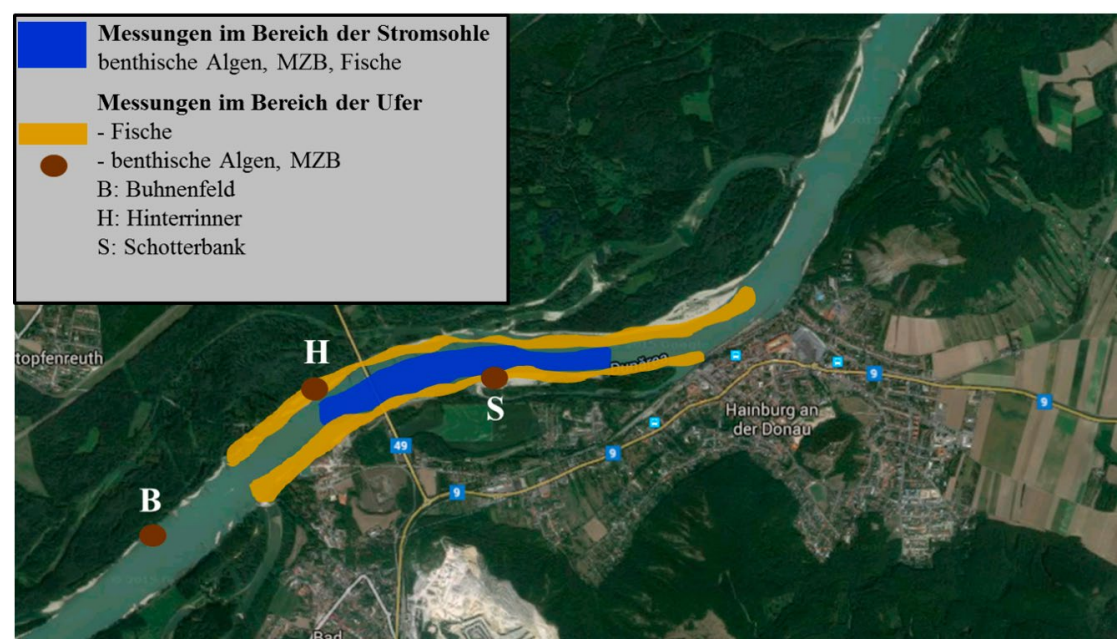

Abb. 2 Ausschnitt aus dem Untersuchungsgebiet und Darstellung der Messbereiche der drei Organismengruppen (Satellitenbild @ Google Earth)

durch Deiche und Dämme, Regulierungsbauwerke und in Folge auch zu Veränderungen der ökologischen Situation (Robertson et al. 2001; Vörösmarty et al. 2010). Diese Veränderungen sind auch an der ganzen Donau, die für die Energieproduktion und als Wasserstraße genutzt wird, zu bemerken (ICPDR 2015).

Betrachtet man diese anthropogenen Veränderungen auf Flussabschnittsebene speziell in Flüssen, die auch als Wasserstraßen dienen, so umfassen diese
Änderungen der Morphologie wie zum Beispiel Begradigungen, Sicherungsund Stabilisierungsmaßnahmen (Schifffahrtsrinne, Uferstabilisierungen, Entfernung von natürlichen Schotterbänken, Einbau von Buhnen) (Sommerwerk et al. 2009) und eine Veränderung des Geschiebetriebs mit dem Resultat einer Eintiefungstendenz des Flussbettes (Tögel et al. 2016, in dieser Ausgabe). Durch diese Veränderungen gehen aber viele bioaktive Zonen und
Retentionsbereiche, die für natürliche Ökosystemfunktionen (z. B. Nährstoffumsatz und -rückhalt) (Hein et al. 2015) wesentlich sind, verloren oder werden beeinträchtigt, was wiederum zu einer Verringerung der Nutzbarkeit von Ökosystemdienstleistungen führen kann.

Flüsse benötigen für eine langfristige ökologische Stabilität diese bioaktiven Zonen als Teil der Flusslandschaft (Schiemer et al. 2001), die bei verschiedenen Abflussbereichen als hoch produktive Lebensräume verfügbar sind und entsprechend mit dem Fluss in Austausch stehen. Diese Bereiche sind wichtig für die flusseigene Tier- und Pflanzenwelt als Refugialräume (z. B. Makrozoobenthos, Rempel et al. 1999; und Fische, Schiemer \& Waidbacher 1992; Rakowitz et al. 2014) oder bestimmte Entwicklungsstadien (Keckeis 2014), dienen benthischen Algengemeinschaften zum Aufbau von Biomasse und sind damit Grundlage für das Nahrungsnetz des Flusses sowie den Stoffrückhalt und Umbau (z. B. Nährstoffaufnahme) (Hein et al. 2005). Entsprechend dem Abflussregime und dem Durchfluss, befinden sich die bioaktiven Zonen (auch als Retentionsbereiche bezeichnet) bei Hochwasserereignissen in den angrenzenden Aulandschaften und Überschwemmungsgebieten, bei Mittelwasser in den dynamischen Seitenarmen und bei Niederwasser entlang der Uferstrukturen, aber auch im Bereich der Stromsohle (Pusch und Fischer 2006) (Abb. 1). Strukturreiche Ufer und die Konnektivität zwischen verschiedenen Lebensraumtypen sind daher wichtig für rheophile Organismen (z. B. Fische), aber auch für den kontinuierlichen Ablauf von Stoffkreisläufen, der Primärproduktion und von Abbauprozessen.

Ein grundlegendes Verständnis der Abläufe und funktionalen Zusammenhänge ist erforderlich, damit im Rahmen von wasserbaulichen Maßnahmen negative Konsequenzen hintangehalten werden können bzw. der ökologische Zustand wieder verbessert werden kann. Im Rahmen des Pilotprojekts Bad Deutsch-Altenburg ist daher nicht nur ein Monitoring der flussbaulichen Maßnahmenumsetzung erforderlich (Tögel et al. 2016, in dieser Ausgabe), sondern auch ein wissenschaftlicher Erkenntnisgewinn über grundlegende Zusammenhänge und biologische Abläufe in unterschiedlichen Habitaten höchst relevant. In diesem Artikel werden Ergebnisse mehrjähriger Forschungsarbeiten in den Tiefenzonen der Donau und typischen 


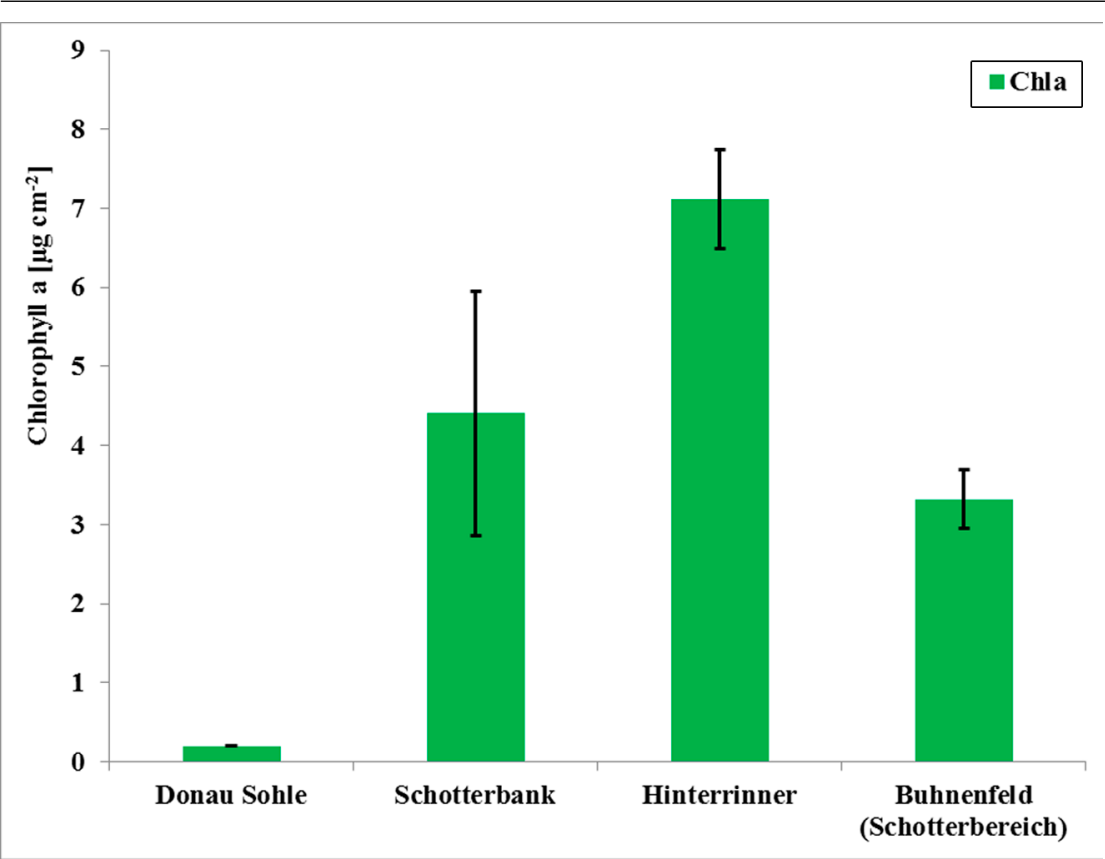

Abb. 3 Benthische Algenbiomasse (Chl-a) an 4 verschiedenen Standorten im Juni 2014. In den unterschiedlichen Uferbereichen wurden jeweils 5-10 Proben entnommen. Mittelwert und Standardabweichung sind dargestellt. *Donau Sohle wurde im März 2015 beprobt

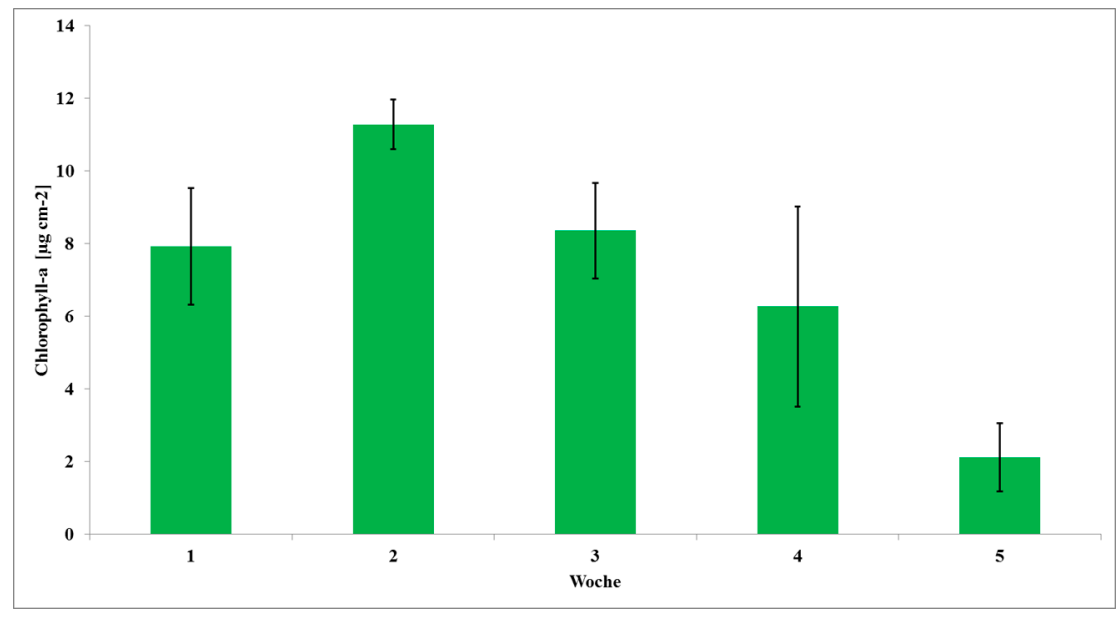

Abb. 4 Benthische Algenbiomasse (Chl-a) gemessen in einer Tiefe von $20 \mathrm{~cm}$ am Standort Hinterrinner im Juli/August 2015. Mittelwert und Standardabweichung von je 5 Proben sind dargestellt

Uferzonenlebensräumen präsentiert, die auch durch die Regulierung veränderte Uferstrukturen inkludiert. Es wird dabei die Frage untersucht, welche Faktoren im Rahmen von integrierten wasserbaulichen Maßnahmen von Bedeutung für die Qualität dieser Lebensräume sind. Es werden Ergebnisse von wichtigen trophischen Niveaus $\mathrm{zu}$ benthischen Algen, zur benthischen Evertebratenfauna (Makrozoobenthos) und zur Fischfauna gezeigt, die die Be-
Verluste an Habitatqualitätund Reduktion von Ökosystemfunktionen (Reckendorfer et al. 2005). Im Zuge des Pilotprojekts Bad Deutsch-Altenburg wurden daher unterschiedliche wasserbauliche Maßnahmen gesetzt, um Verfahren zu entwickeln, die einen Ausgleich der geomorphologischen Defizite ermöglichen (Tögel et al. 2016, in dieser Ausgabe), und die Effekte mittels wissenschaftlichen Monitorings $\mathrm{zu}$ dokumentieren. Im Bereich des Uferrückbaus sind Vergleiche zwischen unterschiedlichen Ufertypen naheliegend. Daher wurden zum einen naturnahe Bereiche wie die Schotterbank (rechtes Donauufer), oder wasserbaulich geschaffene Bereiche wie ein Buhnenfeld (linkes Donauufer) oder neu geschaffene Strukturen wie ein Hinterrinner (= wellengeschütztes, kontinuierlich durchströmtes Seitengerinne hinter einer (aufgeschütteten) Insel) (Simons et al. 2001), ausgewählt (Abb. 2).

\subsection{Benthische Algenuntersuchungen}

Im Juni 2014 wurden flächendeckende Messungen an 3 Uferbereichen Hinterrinner, Buhnenfeld, Schotterbank (Abb. 2) durchgeführt, um die benthische Algenbiomasse $\mathrm{zu}$ bestimmen. Weiters wurden im März 2015 mittels Airliftprobenahme (Fesl et al. 2005) die benthischen Algenbiomassen der Donausohle beprobt. Um die benthische Algenbiomasseentwicklung und Algenartenzusammensetzung in einer ausgewählten Uferzone im Detail zu untersuchen, wurde ebenfalls im Sommer 2015 der Bereich des Hinterrinners 5 Mal im wöchentlichen Abstand beprobt.

An jeder Probenstelle wurden mindestens 5 Steine entnommen und je eine Fläche von $15 \mathrm{~cm}^{2}$ mittels Skalpell abgeschabt. Die Proben wurden sofort gekühlt und im Labor mittels Acetonaufschluss der Chlorophyll-a-Gehalt (Steinman et al. 2011) fluorometrisch bestimmt. Eine Teilprobe wurde für die Pigmentanalyse an einer HPLC (Steinman et al. 2011) entnommen, um die Algengroßgruppenzusammensetzung zu bestimmen. sohle eines großen Flusses für diese Organismengruppen aufzeigen.

\section{Methodik}

Die Donau östlich von Wien ist geprägt durch reduzierte geomorphologische Dynamik, Degradierung des Flussbetts, Isolierung von Nebenarmen und Augewässern und strukturelle Defizite im Hauptstrom und damit einhergehende

\subsection{Makrozoobenthos}

Das Makrozoobenthos wurde in den Jahren 2006 (Voruntersuchung) und 2015 (Nachuntersuchung) in den Uferbereichen (ca. $30 \mathrm{~cm}$ Wassertiefe) und an der Gewässersohle im Bereich der Schifffahrtsrinne jeweils im durch die Maßnahmen beeinflussten Projektgebiet und 


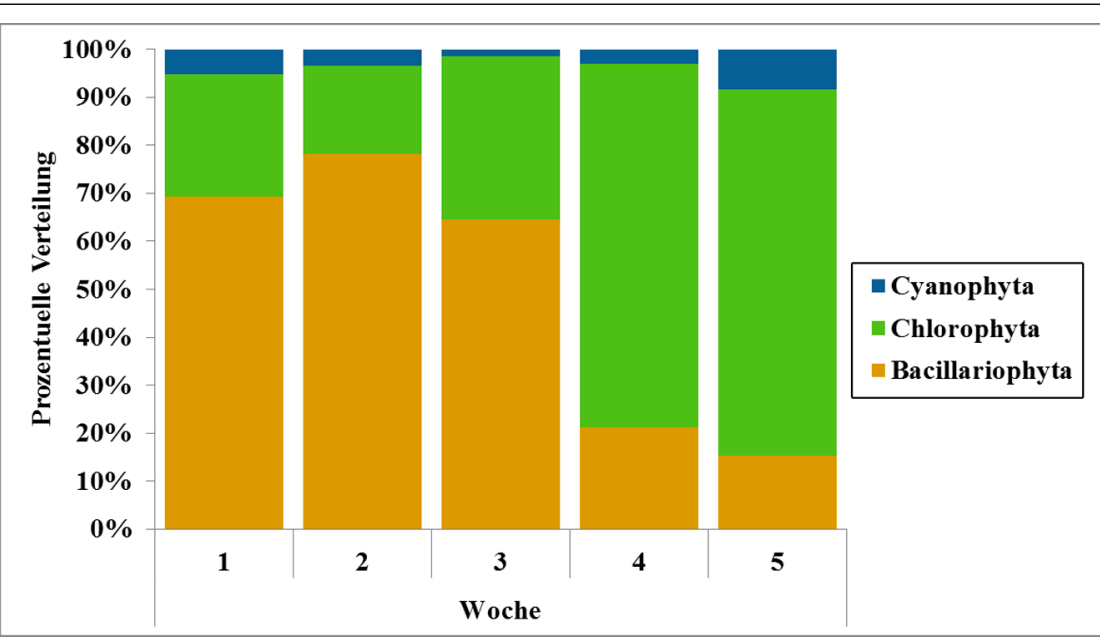

Abb. 5 Prozentuelle Verteilung der Algengroßgruppen Bacillariophyta (Kieselalgen), Chlorophyta (Grünalgen) und Cyanophyta (Blaualgen) in einer Tiefe von $20 \mathrm{~cm}$ am Standort Hintterrinner im Juli/August 2015. Dargestellt ist der Mittelwert von je 5 Proben

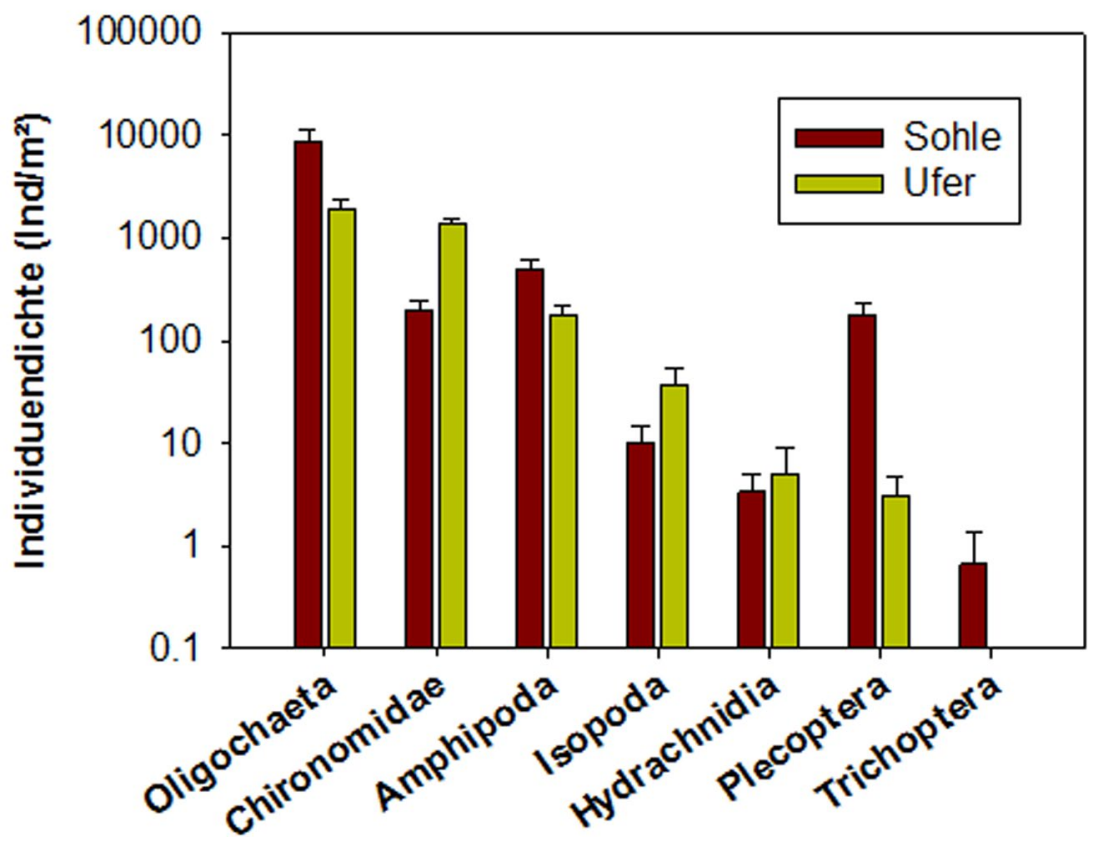

Abb. 6 Abundanzen (Mittelwert und Standardfehler) der sieben häufigsten Großgruppen des MZB im Jahr 2015 an den Ufern und der Gewässersohle der Donau. In beiden Habitaten wurden schottrige Bereiche beprobt. $N=56$

in einer unbeeinflussten Referenzstrecke untersucht. Dabei wurden 201520 Proben am Schotterufer sowie 36 Proben an der Oberfläche und 31 im Interstitial der Sohle entnommen (Abb. 2). Die Proben wurden im März 2015 genommen. Die Proben im Uferbereich wurden auf Schotter mittels Hesssampler (Besammlungsfläche $4,9 \mathrm{dm}^{2}$ ) genommen. Die Stromsohle wurde an der Oberfläche mittels Airliftsampler (Besammlungsfläche 4,1 $\mathrm{dm}^{2}$ ) und in der Tiefe mittels FreezeCore gewässer erfordert den Einsatz und die Kombination verschiedener fischereilicher Methoden (Loisl et al. 2014). Um die Effekte von Maßnahmen abschätzen zu können, erfolgten die Untersuchungen im Projektgebiet in Bad Deutsch Altenburg (Gebiet, in dem Veränderungen erfolgten) und in einer Kontrollstrecke (Gebiet ohne flussbauliche Eingriffe), jeweils vor (2006/2007) und nach (2014/2015) den gewässerbaulichen Maßnahmen. Fischlarven (Größenbereich ca. 0,4-5,0 cm) wurden mit einer standardisierten Kescherfangmethode („Point-Abundance-Sampling“, PAS) und mittels eines Uferzugnetzes im unmittelbaren Uferbereich und größere Jungfische $(>5 \mathrm{~cm})$ mittels Elektrobefischungen mit der Handanode besammelt. Die benthische bzw. stromsohlennahe Fischgemeinschaft wurde mittels Langleinenbefischungen untersucht. Adulte Individuen wurden mittels Elektrobefischungen mit Rechenanode vom Boot aus gesammelt. Abbildung 2 zeigt beispielhaft die räumliche Anordnung der Probenbereiche des fischökologischen Monitorings. Um repräsentative Ergebnisse zu erzielen, erfolgten die Proben in 14-tägigen bis monatlichen Intervallen und erstreckten sich zeitlich über alle Saisonen eines Jahreszyklus. Im Jahr 2014 wurden z. B. 955 Mikrohabitate mittels PAS besammelt, 569 Probennahmen erfolgten mittels Ufer-Elektrobefischungen, 37 Langleinen wurden gesetzt (weitere $36 \mathrm{im} \mathrm{Jahr} \mathrm{2015)}$ und 319 Elektrobefischungen erfolgten mittels Rechenanode vom Boot aus. Die Untersuchungen mittels Echolot erfolgten vier Mal pro Jahr über jeweils 24 Stunden, um die saisonale und diurnale Aktivität der Fische zu untersuchen.

\section{Ergebnisse und Diskussion}

\subsection{Benthische Algen}

Als Grundlage für alle weiteren trophischen Ebenen dienen benthische Algen, die mittels Photosynthese Biomasse aufbauen. Die Daten zeigen, dass an der Stromsohle die geringste Biomasse gebildet wurde. Werte von $3,1 \pm 0,9 \mu \mathrm{g} \mathrm{cm}^{-2}$ wurden am Schotterbereich der Buhne gemessen. Der höchste Wert mit bis zu 7,1 $\pm 0,6 \mu \mathrm{g} \mathrm{cm}^{-2}$ wurden im Hinterrinner erreicht. Konstante Werte $\left(4,4 \pm 1,5 \mu \mathrm{g} \mathrm{cm}^{-2}\right)$ wurde an der rechtsufrigen Schotterbank gemessen (Abb. 3). Somit waren der Hinterrinner, die natürliche Schotterbank als auch der Schotterbereich im Buhnenfeld wichtige Bereiche 


\section{Sohle 2015}

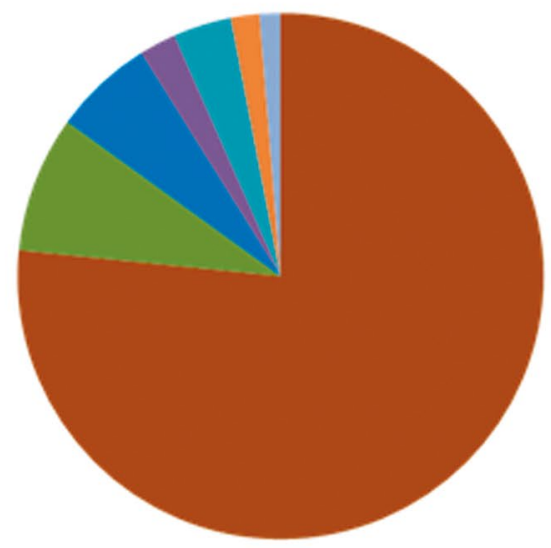

Ufer 2015

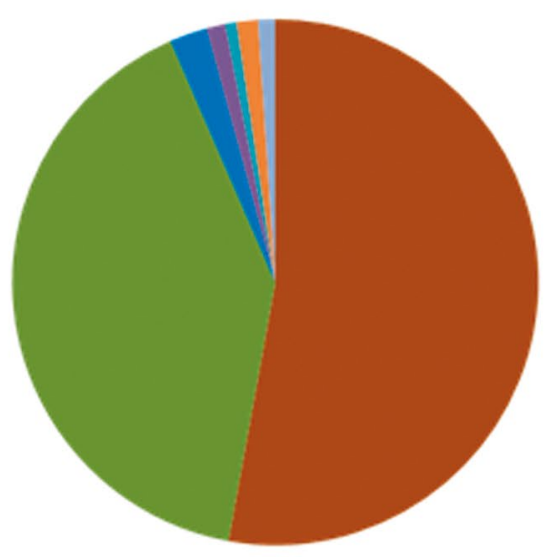

Detritivora

Weidegänger

Räuber

Sonstige

passive Filtrierer

Zerkleinerer

aktive Filtrierer

Abb. 7 Mittlere Verteilung der Fraßgruppen des MZB (nach Moog 2002) im Jahr 2015 in den Ufern und der Gewässersohle der Donau

für benthische Biomasseentwicklung und Primärproduktion, was wiederum einen großen Einfluss auf die höheren trophischen Ebenen haben kann.

Um einen genaueren Einblick in die Dynamik der Besiedelung einer Uferzone mit benthischen Algen zu bekommen, wurde der Standort Hinterrinner intensiver untersucht. Die Besiedelung und Biomasseentwicklung während einer Niederwasserperiode im Sommer 2015 sind daher im Folgenden dargestellt. Die Ergebnisse zeigen zum einen, dass die Proben eine hohe Standardabweichung aufwiesen, was darauf schließen lässt, dass die benthischen Algen sehr heterogen am Schotter verteilt waren (Abb. 4). Zum anderen kann man erkennen, dass es im zeitlichen Verlauf von Woche $1 \mathrm{zu}$ Woche $2 \mathrm{zu}$ einem Anstieg und dann in weiterer Folge zu einem Abfall der Algenbiomasse kam.

Betrachtet man die prozentuelle Verteilung der Algengroßgruppen Bacillariophyta (Kieselalgen), Chlorophyta (Grünalgen) und Cyanobakterien (Blaualgen), so kann man erkennen, dass die Algengemeinschaft des Hinterrinners am Beginn der Untersuchung eine Kieselalgen-dominierte Gesellschaft war (Abb. 5).

Nachdem der Pegel kontinuierlich abgesunken war (Woche 4), dominierte die Gruppe der Chlorophyta. Das Vorherrschen einer Grünalgen-dominierten Gesellschaft wurde auch bei der Voruntersuchung gezeigt, allerdings schon bei Wasserständen knapp unter Mittelwasser, da es hier im Buhnenbereich zu großen Flächen ohne Anbindung mit der Donau kam (44\% der Buhne wiesen Rückstaubereiche auf - nach Bau des Hinterrinners waren es nur mehr $22 \%$ ).

Zusammenfassend zeigen die Ergebnisse, dass eine permanente Strömung im Bereich des Hinterrinners eine Besiedelung einer flusstypischen Kieselalgen dominierten Algengemeinschaft fördert und hohe Biomassen erreicht werden können. Erst bei längeren Niederwasserphasen kann es zu einem Rückgang der Kieselalgen und zu einem Anstieg der Grünalgen kommen. Somit zeigen diese Ergebnisse, dass nach der Umstrukturierung der Uferzonen zu einem Hinterrinner die sich entwickelnde Algenbiomasse höheren Flussorganismen in dem gut verbundenen Lebensraum über einen längeren Zeitraum zur Verfügung stehen kann. Um die Wirksamkeit des Hinterrinners als ökologisch wertvolles Habitat im Detail zu kennen, werden noch genauere Analysen vorgenommen. Dies kann als Grundlage für weitere Maßnahmen dienen. Diese Uferstrukturen bieten eine wesentliche Grundlage für die Versorgung des flusseigenen Nahrungsnetzes.

\subsection{Makrozoobenthos (MZB)}

Eine Organismengruppe, die sehr stark auf die Nahrungsverfügbarkeit und daher auch auf die benthischen Algen im und am Sediment angewiesen ist und auf Substratbedingungen reagiert, stellt das Makrozoobenthos dar. Vergleicht man die Habitattypen schottrige Ufer und Stromsohle, so sind deutliche Unterschiede in den Besiedlung durch das Makrozoobenthos zu erkennen (Abb. 6). In beiden Habitaten
(Schotter im Bereich von Ufer und Sohle) dominierten die Oligochaeten das MZB, wobei sie auf der Stromsohle höhere Dichten erreichten als an den Ufern, die Chironomiden hingegen erreichten höhere Dichten am Ufer als auf der Sohle.

Die Unterschiede in der Zusammensetzung der Großtaxa und Arten spiegelte sich auch in der Zusammensetzung der funktionellen Gruppen wieder (Abb. 7) In den Habitaten mit groben Substrat (Schotter im Bereich der Sohle) dominierten die Detritusfresser. Im Bereich der Schotterufer spielten die Weidegänger ebenso eine große Rolle. Dieser Trend war besonders im Jahr 2015 deutlich. Der höhere Anteil an Weidegängern lässt vor allem auf die größere Verfügbarkeit von benthischen Algen in den seichten Wasserbereichen schließen.

Neben den Uferzonen ist auch die Donausohle ein wichtiger Lebensraum und Refugialraum (Stubbington 2012) für das Makrozoobenthos. Im Interstitial (= Lückenraum des Sediments) der Sohle wurden nennenswerte Besiedlungsdichten bis in Tiefen von 1 Meter im Sediment gefunden (Abb. 8). Auch die Besiedlung des Interstitials zeigte sehr große Unterschiede zwischen den und Variabilität innerhalb der Großgruppen. Die Oligochaeten erreichten deutlich höhere Dichten in oberflächennahen Bereichen zeigen aber auch hohe Dichten in größeren Tiefen. Bei den Chironominden kann eine sehr ähnliche Besiedlungsstruktur beobachtet werden, jedoch mit höchsten Dichten in etwas tieferen Schichten und einer deutlichen Abnahme mit der Tiefe. Vor 
Oligochaeta

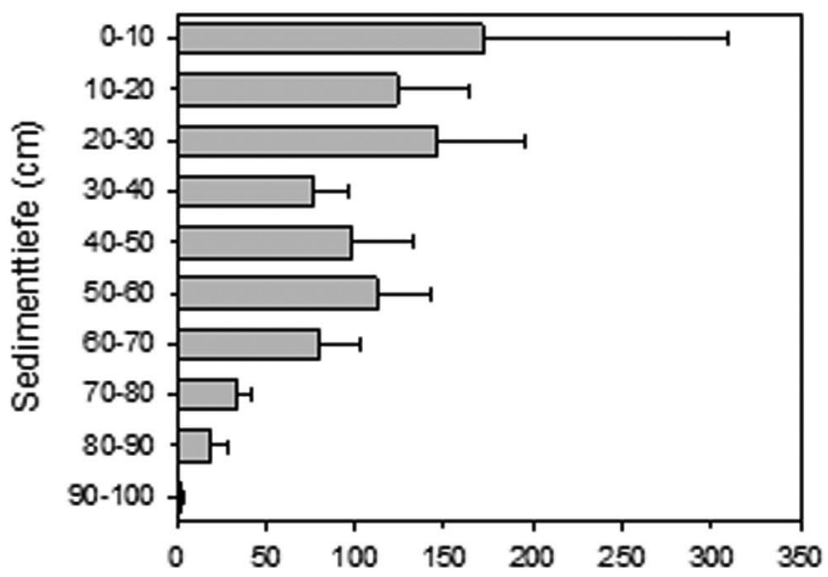

Amphipoda

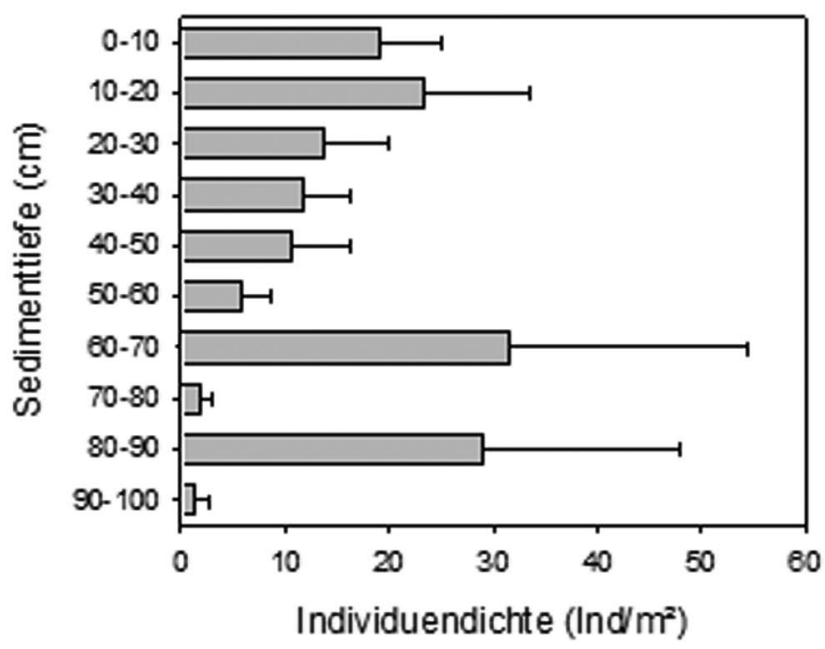

\section{Chironomidae}

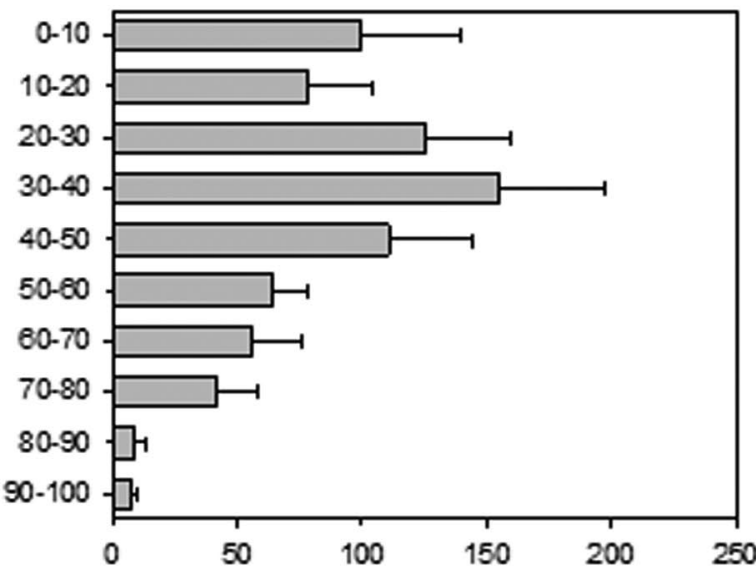

Isopoda

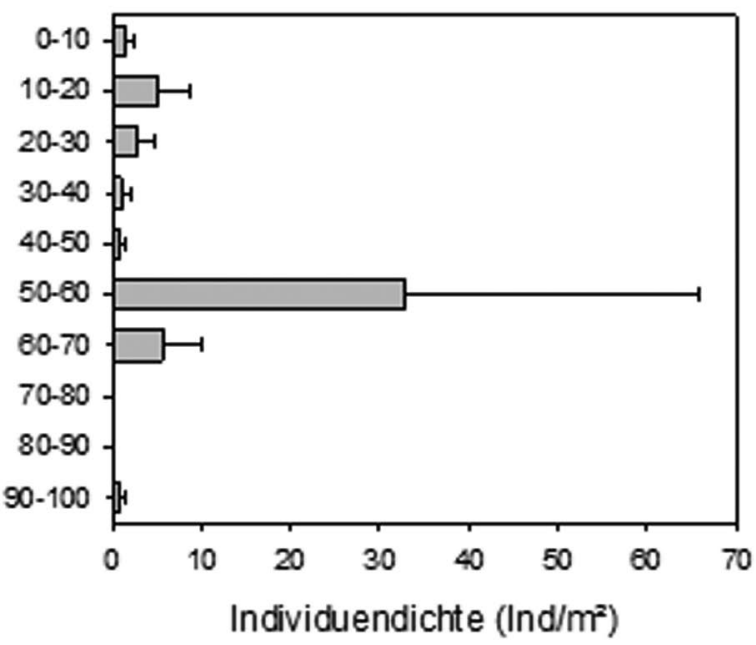

Abb. 8 Tiefenbesiedlung im Interstitial der vier häufigsten Großgruppen des MZB im Jahr 2015 (Mittelwert und Standardfehler)

allem die Amphipoden aber auch die Isopoden erreichten im Jahr 2015 hohe Besiedlungsdichten in höheren Tiefen (50 bis $90 \mathrm{~cm})$. Auch im Vergleich zur Voruntersuchung gibt es deutliche Unterschiede in den Besiedlungstiefen und Dichten, die aber vorrangig sowohl im Bereich der Maßnahme (Bereich der eingebrachten Granulometrischen Sohlverbesserung - GSV), (Gmeiner et al. 2016, in dieser Ausgabe) als auch in unbeeinflussten Bereichen (Referenzstrecke) zu beobachten sind und demnach im natürlichen Schwankungsbereich des Systems liegen und nicht auf die Maßnahme selbst zurückgeführt werden können. Um die Auswirkungen der Maßnahme im Detail zu kennen, sind jedoch noch genauere Analysen notwendig.

\subsection{Fischzönose}

Die Bedeutung von bestimmten Uferzonen im Hauptgerinne der Donau für die Jungfischgemeinschaft wurde schon von Schiemer und Spindler (1989) untersucht, wobei erstmals die Bindung von den frühen Entwicklungsstadien von Fischen (,breeding and fry stage") an Habitate des Hauptgerinnes beschrieben und analysiert wurde. Diese Arbeiten zeigten deutlich, dass die charakteristischen Fischarten nicht nur, wie früher angenommen, in Zuflüssen der Donau, sondern auch im Hauptgerinne reproduzieren, und dass die Jungfische vieler gefährdeter Arten Uferhabitate im Hauptstrom bevorzugen bzw. unmittelbar auf diese angewiesen sind.
Die Ergebnisse des fischökologischen Monitorings zeigen deutlich, dass die Ufer auch von älteren Entwicklungsstadien (juvenile und subadulte Stadien) vieler Arten besiedelt werden. Abbildung 9 zeigt einen Vergleich der Arten-Areal-Kurve der Stichproben der Uferfänge mit jenen der Proben im Sublitoral (tiefere Bereiche am Rand der Schifffahrtsrinne). Es zeigt sich, dass die Artenzahl in Uferzonen bereits mit wenigen Stichproben steiler ansteigt (mit jeder Stichprobe werden mehrere neue Arten gefangen) als jene im Sublitoral, und mit ca. 100 Stichproben eine Verflachung der Kurve zu verzeichnen ist (Sättigung, ein Großteil der Vorkommenden Arten wurde erfasst). Die erreichte Gesamtartenzahl ist mit 39 Arten beim Ufer höher als jene im Sublitoral (35 Arten). 


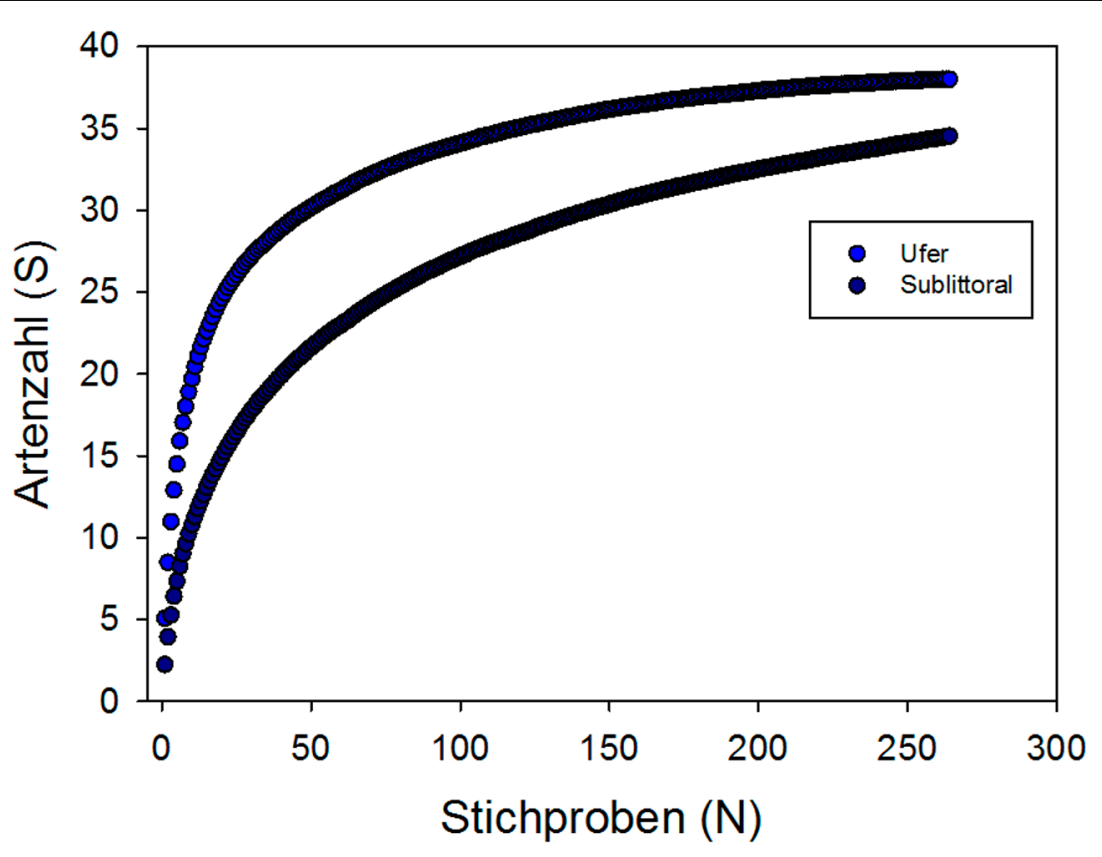

Abb. 9 Vergleich der Arten-Areal-Kurven der Stichproben im Uferbereich (Befischung mit Handanode) und in anschließenden, tieferen Bereichen im Sublitoral des Hauptgerinnes der Donau (Bereich Bad Deutsch-Altenburg und Hainburg, Vergleichszeitraum 2006/2007 und 2014)

Der Vergleich der durchschnittlichen Artenzahl pro Probe sowie des Biodiversitätsindex (Shannon-Wiener-Index) zeigt für beide Variablen signifikante Unterschiede zwischen den beiden Gewässerbereichen an (Mann-Whitney-U-Test, NUfer $=228 ; \quad$ NSublittoral $=535 ; \quad p<0,001$ ) (Abb. 10). Durchschnittlich werden in einer Stichprobe am Ufer 5, und im Sublitoral 2 Fischarten erfasst, Der Schwankungsgereich der Werte ist ähnlich, im Uferbereich werden zwischen 0 und 17 Arten in einer Probe gefangen, im Sublitoral zwischen 0 und 16 Arten. Dies kann dadurch bedingt sein, dass jüngere und kleinere Individuen derselben Arten die Uferbereiche und die älteren, größeren Individuen die tieferen Gewässerbereiche nutzen. Insgesamt ist aber eine höhere Biodiversität in den Ufern vorzufinden, was in diesem Fall nicht auf eine unterschiedliche Artenzahl, sondern auf unterschiedliche Abundanzen einzelner Arten in den beiden Gewässerbereichen zurückzuführen ist.

Die Uferzonen in der Donau dienen den frühen Entwicklungsstadien als Aufwuchshabitat und werden von Jungfischen (juvenilen Stadien) vieler charakteristischen Fischarten bevorzugt genutzt und besiedelt. Diese Gewässerbereiche weisen in den mehrjährigen Untersuchungen die höchste Biodiversitätswerte der Fischgemeinschaft auf. Im Zuge vongewässerbaulichen Maßnahmen mögliche Effekte auf die Zönosen gerichtet werden. Erste Hinweise und Erkenntnisse auf die Auswirkungen von Uferrevitalisierungsmaßnahmen auf die Fischgemeinschaft konnten im Bereich Witzelsdorf gewonnen werden (Keckeis et al. 2014). Nach der Entfernung des Blockwurfes und der Errichtung neuer Buhnen entwickelte sich ein naturnahes Ufer, mit entsprechender morphologischer Diversität, Umlagerungsprozessen und einer gleichförmigen, geringen Strömung entlang des gesamten Uferbereiches. Die fischökologischen Untersuchungen zeigten, dass nach diesen Maßnahmen eine sehr ausgeprägte Veränderung in der Struktur der Fischartengemeinschaft erkennbar war. Vor allem die Bestände der invasiven Arten (Schwarzmundgrundel, Kesslergrundel) nahmen deutlich ab, und in den folgenden Jahren nahmen die charakteristischen Arten der rheophilen Gilde bzw. jener der eurytopen Gilde deutlich zu (Abb. 11).

\section{Zusammenfassung}

Flüsse benötigen für eine langfristige ökologische Stabilität Bereiche, die bei verschiedenen Abflusssituationen als hoch produktive Lebensräume verfügbar sind und entsprechend mit dem Fluss in Austauschstehen, diese sind aberin regulierten muss daher höchste Aufmerksamkeit auf
Flüssen meist stark verändert (Schiemer et al. 2001). Diese Retentionsbereiche oder bioaktive Zonen sind wichtig für die flusseigene Tierwelt als Refugialräume (z. B. Makrozoobenthos, Rempel et al. 1999; und Fische, Schiemer und Waidbacher, 1992; Rakowitz et al. 2014) oder bestimmte Entwicklungsstadien (Keckeis 2014), dienen benthischen Algengemeinschaften zum Aufbau von Biomasse und sind damit Grundlage für das Nahrungsnetz des Flusses sowie den Stoffrückhalt und Umbau (z. B. Nährstoffaufnahme) (Hein et al. 2005). Vor allem während Niederwasser sind die Uferbereiche und die Stromsohle selbst von wesentlicher Bedeutung. Im Zuge des Pilotprojektes Bad Deutsch-Altenburg wurden Grundlagen zur Qualität und Besiedelung dieser beiden zentralen Lebensräume im Hinblick auf Maßnahmen in diesen Bereichen untersucht.

Die Stromsohle wies eine verhältnismäßig geringe Biomasse benthischer Algen auf. Wesentliche Nahrungsquelle für das Makrozoobenthos ist daher das transportierte organische Material und die dominierenden Großgruppen wurden von den Oligochaeten und Chironomiden gebildet. Im Interstitial (= Lückenraum des Sediments) der Sohle wurden nennenswerte Besiedlungsdichten des Makrozoobenthos bis in Tiefen von 1 Meter im Sediment gefunden und belegen die Bedeutung dieses Lebensraums und speziell des Interstitials. Auch für Fische ist die Stromsohle ein wichtiges Habitat und wurde vor allem von älteren, größeren Individuen genutzt.

Bei den Uferzonen zeigte sich, dass natürliche Zonen, wie zum Beispiel eine Schotterbank, oder neu geschaffene Bereiche wie ein Hinterrinner eine hohe Biomasse an benthischen Algen mit einer flusstypischen Kieselalgendominierten Gemeinschaft aufweisen kann. Erst bei längeren Niederwasserphasen kann es zu einem Rückgang der Kieselalgen und $\mathrm{zu}$ einem Anstieg der Grünalgen kommen. In diesem Bereich wurde auch ein höherer Anteil an Weidegängern beim Makrozoobenthos ermittelt, was vor allem auf die größere Verfügbarkeit von benthischen Algen in den seichten Wasserbereichen zurückzuführen ist. Ebenfalls zeigte sich, dass die neustrukturierten Uferzonen der Donau den frühen Entwicklungsstadien der Fische als Aufwuchshabitat dienten und von Jungfischen (juvenilen Stadien) vieler charakteristischen Fischarten bevorzugt genutzt und besiedelt wurden. Frühere Studien zeigten auch, dass in 

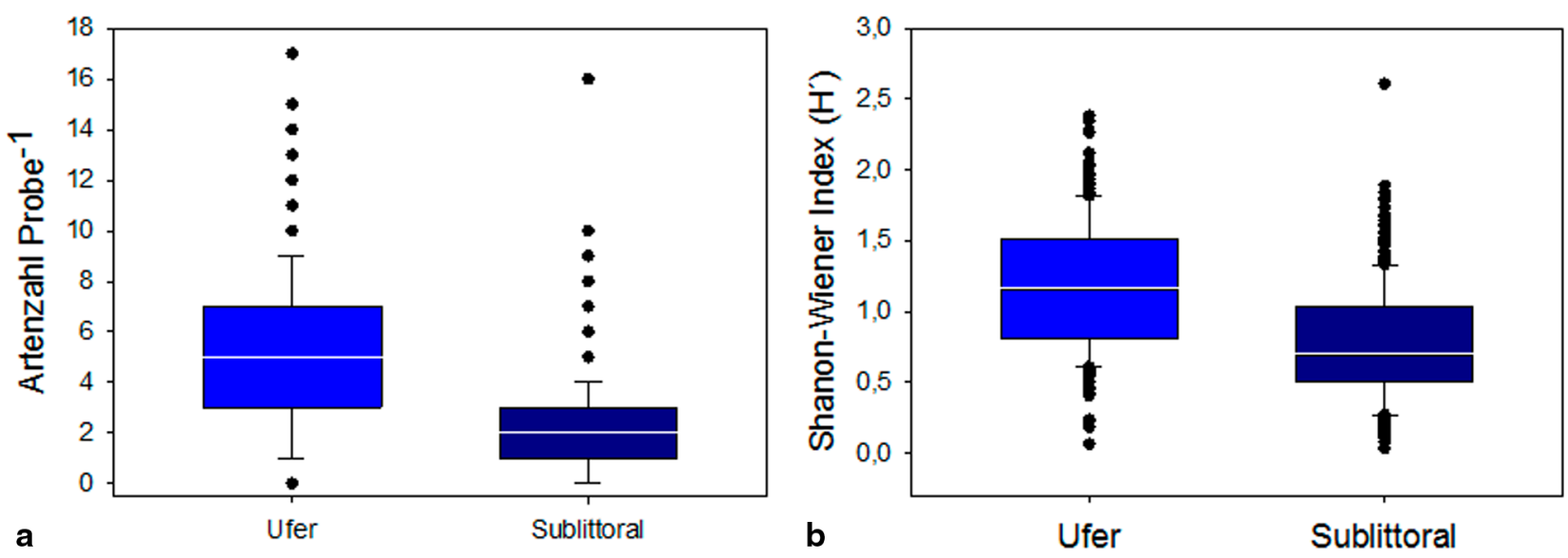

Abb. 10 links: Vergleich der Artenzahl pro Probe in den Uferfängen $(N=228)$ und in den Proben im Sublitoral. Die Unterschiede sind höchst signifikant (Mann-Whitney-U-Test, $N_{\text {Ufer }}=228 ; N_{\text {Sublittoral }}=535 ; p<0,001$ ). rechts: Vergleich des Shannon-Wiener-Diversitätsindex zwischen den beiden Gewässerbereichen. Die Werte der beiden Gewässerbereiche sind höchst signifikant (MannWhitney-U-Test, $\left.\mathrm{N}_{\text {Ufer }}=228 ; \mathrm{N}_{\text {Sublittoral }}=535 ; p<0,001\right)$

2007

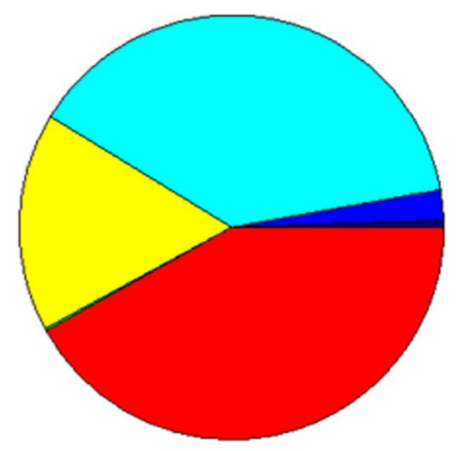

2012

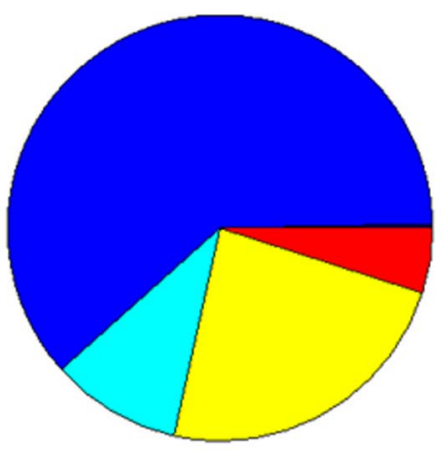

2014

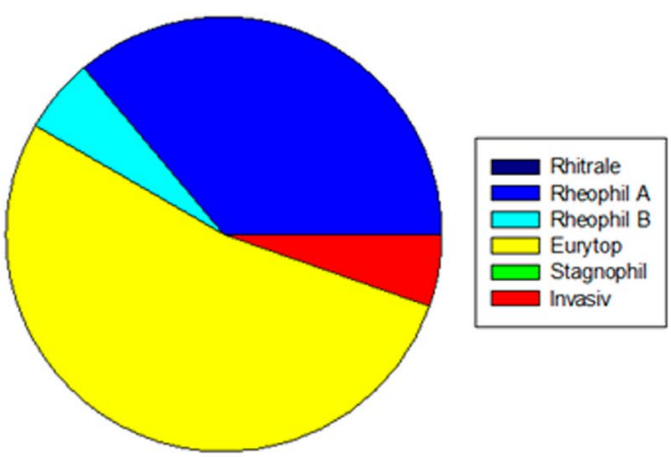

Abb. 11 Prozentuelle Zusammensetzung der ufernahen Fischartengemeinschaft im Bereich der restrukturierten Strecke in Witzelsdorf. Vor den Restaurierungsmaßnahmen im Jahr 2007 wurde die Artengemeinschaft von invasiven Arten (rot, Schwarzmundgrundel, Kesslergrundel) dominiert. Nach den Maßnahmen (2012) dominierten rheophile Arten, der Anteil der invasiven Fischarten ging stark zurück, in weiterer Folge (2014) nahm der Anteil rheophiler Arten etwas ab, und eurytope Arten dominieren die Gemeinschaft. Der Anteil invasiver Arten bleibt konstant auf niedrigem Niveau

den strukturreichen Uferzonen invasive Arten abnahmen und charakteristische, strömungsliebende Arten wieder zunahmen (Keckeis 2014).

Diese ersten Ergebnisse zeigen daher, dass wasserbauliche Maßnahmen im Bereich der Stromsohle (Granulometrische Sohlverbesserung) und der Ufer (Uferrückbau, Niederwasserregulierung) einen großen Einfluss auf die Artverteilung und die Grundlagen des Nahrungsnetzes haben kann. Vor allem durch die Umstrukturierung der Uferzonen ist zu erwarten, dass es zu einer Aufwertung dieser Habitate kommt, und sich wieder verstärkt ein flusstypisches Nahrungsnetz etablieren kann. Die ökologische Qualität und die Nachhaltigkeit dieser Maßnahmen werden in weiterer Folge durch die Nachuntersuchungen des Pilotprojektes evaluiert.

\section{Danksagung}

Das Pilotprojekt Bad Deutsch-Altenburg wird durch das Bundesministerium für Verkehr, Innovation und Technologie finanziert. Weitere finanzielle Mittel werden durch die Europäische Union über die Transeuropäischen Verkehrsnetze (TEN-V) beigetragen.
Open Access Dieser Artikel unterliegt den Bedingungen der Creative Commons Attribution License. Dadurch sind die Nutzung, Verteilung und Reproduktion erlaubt, sofern der/die Originalautor/en und die Quelle angegeben sind.

\section{Einhaltung ethischer Richtlinien}

Interessenkonflikt

Es besteht kein Interessenkonflikt. 


\section{Literatur}

Fesl, C., Humpesch, U.H., \& Wöss, E.R. (2005) Biodiversität des Makrozoobenthos der österreichischen Donau unter Berücksichtigung quantitativer Befunde der Freien Fließstrecke unterhalb Wiens. Denisia 16, 139-158.

Hein, T., Reckendorfer, W., Thorp, J. H., \& Schiemer, F. (2005): The role of slackwater areas for biogeochemical processes in rehabilitated river corridors: examples from the Danube. Arch. Fur Hydrobiol. Suppl. Large Rivers, 15, 425-442.

Hein, T., Schwarz, U., Habersack, H., Nichersu, I., Preiner, S., Willby, N. \& Weigelhofer, G. (2015): Current status and restoration options for floodplain along the Danube River. Sci. Total Environment.

ICPDR (2015): Joint Danube Survey 3 - A comprehensive analysis of Danube water quality. Vienna, $369 \mathrm{pp}$

Keckeis, H. (2014): Short-term effects of inshore restoration measures on early stages, benthic species, and the sublittoral fish assemblage in a large river (Danube, Austria). Hydrobiologia, 729(1), 61-76.

Keckeis, H., Lumesberger-Loisl, F., Reiter, K., Schulze, C.H. (2014): Restrukturierungsmaßnahmen in großen Flüssen: Auswirkungen des Uferrückbaues und der Buhnenoptimierung im Hauptstrom der freifließenden Donau in Witzelsdorf (NÖ) auf die Artengemeinschaft von Indikatororganismen (Vegetation, Vogel- und Fischfauna). Österreichs Fischerei 67. Jahrgang: 57-66

Loisl, F., Singer, G., \& Keckeis, H. (2014) Method-integrated fish assemblage structure at two spatial scales along a free-flowing stretch of the Austrian Danube. Hydrobiologia, 729(1), $77-94$
Moog, O. (Ed.) (2002): Fauna Aquatica Austriaca, Lieferung 2002 - Katalog zur autökologischen Einstufung aquatischer Organismen Österreichs. Wasserwirtschaftskataster, Bundesministerium für Land- und Forstwirtschaft, Umwelt und Wasserwirtschaft, Wien.

Pusch, M. \& Fischer, H.(Hrsg.) (2006): Stoffdynamik und Habitatstruktur in der Elbe Konzepte für die nachhaltige Entwicklung einer Flusslandschaft, Bd. 5, 404 Seiten, ISBN 978-3-89998-011-0.

Reckendorfer, W., Schmalfuss, R., Baumgartner, C., Habersack, H., Hohensinner, S., Jungwirth, M. \& Schiemer, F. (2005): The Integrated River Engineering Project for the Free-flowing Danube in the Austrian Alluvial Zone National Park: contradictory goals and mutual solutions. Arch. Hydrobiol. Suppl. 155/1-4, 613-630.

Rempel, L.L., Richardson, J.S., and Healey, M.C. (1999): Flow refugia for benthic macroinvertebrates during flooding of a large river. J. North Am. Benthol. Soc. 18: 34-48.

Robertson, A.I., Bacon, P. and Heagney, G. (2001): The responses of floodplain primary production to flood frequency and timing. Journal of Applied Ecology, 38: 126-136. doi:10.1046/j.1365-2664.2001.00568.x.

Rakowitz, G., Berger, B., Schludermann, E. Tritthart, M., Habersack, H., \& Keckeis, H. (2014): Deep pools of the Danube River: ecological function or turbulent sink?. Hydrobiologia, 729(1), 143-159.

Schiemer, F., \& Spindler, T. (1989): Endangered fish species of the Danube River in Austria. Regulated Rivers: Research \& Management, 4(4), 397-407.

Schiemer, F., \& Waidbacher, H. (1992): Strategies for conservation of a Danubian fish fauna. River conservation and management, 26, 363-382.
Schiemer, F., Baumgartner, C. \& Tockner, K. (1999): Restoration of floodplain rivers: the 'Danube restoration project'. Regulated Rivers: Research and Management, 15, 231-244. Simons, H.E.J., Bakker, C., Schropp, M.H.I., Jans, L.H., Kok, F.R. \& Grift, R.E. (2001): Man-made secondary channels along the River Rhine (the Netherlands): results of post-project monitoring Regulated Rivers: Research and Management, 17, 473-491.

Sommerwerk, N., Hein, T., Schneider-Jacoby, $M$. Baumgartner, C., Ostojic, A., Siber, R., Bloesch, J., Paunovic, M., Tockner, K., (2009): The Danube River Basin. - In: Tockner K, Uehlinger U, Robinson CT, Rivers of Europe. Amsterdam: Academic Press, pp 59-113.

Steinmann, A.D., Lamberti, G.A., \& Leavitt, P.R., (2011): Biomass and Pigments of benthic Algae in Methods in Stream Ecology Hauer F.R. Lamberti G.A. (Eds) Academic Press, 27.04.2011 - 896 Seiten.

Stubbington, R. (2012): The hyporheic zone as an invertebrate refuge: a review of variability in space, time, taxa and behaviour. Marine and Freshwater Research, 63, 293-311.

Winiwarter, $V_{0}$ Schmid, M., Hohensinner, S. \& Haidvogl, G. (2013): „The environmental history of the Danube River Basin as an issue of long-term socio-ecological research“. In Long Term SocioEcological Research, 103-22. Springer Netherla nds. Österr Wasser- und Abfallw 2016 • 68:239-247 